\title{
Correcting the pandemic: Analysis of corrections to journal articles on COVID-19 and Ebola
}

\section{Jakov Matas, Ivan Buljan, Ana Marušić}

Department of Research in Biomedicine and Health, University of Split School of Medicine, Split, Croatia

\begin{abstract}
OVID-19, declared by the World Health Organization (WHO) as public health emergency of international concern on 30 January 2020 and as pandemic on 12 March 2020 [1], has brought unprecedented interest in research about this disease, resulting in massive publication output and shortened time to publish [2]. The need for rapid communication of research puts pressure on journals to balance rigorous review process with publication speed. Recent retractions in two major medical journals [3] have put doubt on the ability of journals to deal with these pressures. Some journals have already warned about the quality of evidence on COVID-19 [4] and there is concern that poor quality research on COVID-19 presents research waste and prevents pandemic response based on evidence [5].
\end{abstract}

We analyzed corrections to COVID-19 literature in the first five months of 2020, and compared them to those on Ebola, a recent public health emergency of international concern [6]. We searched PubMed using terms "COVID-19" (1 January-31 May 2020) and "Ebola" (1 August-31 December 2014) to retrieve relevant publications and their corrections in five months around the declaration of public emergency. We also searched CrossRef database for corrections to the retrieved articles, and Retraction Watch database (https://retractionwatch.com/retracted-coronavirus-covid-19-papers/). Two authors checked the correction/retractions notices and relevant articles in PubMed and at journals' websites. The search strategy and the list of identified corrections and retractions are available in OSF (https://osf.io/nkp4t).

In the five months surrounding the declaration of a global public health emergency, there were 18863 published items for COVID-19 and 962 for Ebola (Table 1). We identified 78 corrected or retracted articles on COVID-19 (0.4\%) and 7 corrected articles on Ebola (0.7\%). Most of them were research articles, and the corresponding authors mostly came from Asia for COVID-19 articles. Corrections to the articles on COVID-19 were published in 57 different journals and in 6 journals on Ebola. The impact factor of journals publishing corrections to COVID-19 articles was higher than those publishing articles on Ebola (median $=9.193,95 \%$ confidence interval $(\mathrm{CI})=5.873-19.315$ vs $3.570,95 \% \mathrm{CI}=1.817$ to 27.604 ).

The median time between article publication and its correction was 22 days (95\% CI=15-27) for COVID-19 and 82 days (95\% CI=1-87) for Ebola. Reasons for corrections were mostly changes to data (56 for COVID-19 and 10 on Ebola), predominantly non-numerical (36 for COVID-19, none for Ebola) (Table 1). 24 of the corrected or retracted COVID-19 articles included patients (median number of patients $=32,95 \% \mathrm{CI}=4-275$ ). There were no studies involving patients among corrected articles on Ebola. Most of the corrections notices were signed by the authors ( $\mathrm{n}=35$ for articles on COVID-19 and $\mathrm{n}=3$ for articles on Ebola) or there was no statement ( $n=42$ and $n=4$, respectively); in 3 cases for COVID-19 ar- 
ticles a journal signed the statement. Corrected articles for both topics were mostly adequately indexed in PubMed: 71 were "published erratum" for articles on COVID-19 and 7 for those on Ebola. For articles on COVID-19 there were 5 "retractions of publication", 1 "addendum" and for 3 articles the corrections were not indexed as such (one correction from a journals was not indexed, and in two cases the original articles were not indexed but the indexed version had "retracted" or "withdrawn" in the title.

Table 1. Corrections of articles published about COVID-10 $(n=18863)$ and Ebola $(n=962) *$

\begin{tabular}{|c|c|c|}
\hline Characteristics & COVID-19 & EвоLA \\
\hline Number of corrected articles (\% of total published articles) & $78(0.4)$ & $7(0.7)$ \\
\hline Number of corrections/retractions $\dagger$ & $73 / 7$ & $7 / 0$ \\
\hline \multicolumn{3}{|l|}{ Type of corrected/retracted articles: } \\
\hline Research article/systematic review & 33 & 2 \\
\hline Review article & 11 & 0 \\
\hline Other (letter to the editor, commentaries, opinion pieces, reports, etc.) & 34 & 5 \\
\hline \multicolumn{3}{|c|}{ Geographical origin of corrected/retracted articles (corresponding author address): $\ddagger$} \\
\hline Europe & 24 & 2 \\
\hline Asia and Australia & 33 & 1 \\
\hline Africa & 1 & 1 \\
\hline South America & 1 & 0 \\
\hline North America & 19 & 3 \\
\hline \multicolumn{3}{|l|}{ Reasons for correction: } \\
\hline Data (including figures and tables)§ & 53 & 6 \\
\hline Bibliographical information & 12 & 1 \\
\hline Authorship & 4 & 0 \\
\hline Combination of above & 4 & 0 \\
\hline \multicolumn{3}{|l|}{ Reasons for retraction: } \\
\hline Data (including figures and tables)II & 5 & 0 \\
\hline Not stated & 2 & 0 \\
\hline
\end{tabular}

*Time-frame for the articles: COVID-19: 1 January 2020 to 31 May 2020, Ebola: 1 August 2014 to 31 December 2014. $\dagger$ One COVID-19 article was first corrected and then retracted; and one was corrected twice.

$\$ 21$ articles on COVID-19 originated from China; USA was the country of origin for 18 COVID-19 articles and all three Ebola articles.

§Reasons for data correction: change in numerical data ( $n=13$ for COVID-19), change in non-numerical data ( $\mathrm{n}=35$ for COVID-19, 6 for Ebola), both numerical and non-numerical data ( $\mathrm{n}=4$ for COVID-19), not clear ( $\mathrm{n}=1$ for COVID-19).

TIReasons for data retractions: authors falsely claiming first-hand experience $(n=1)$, incorrect data calculation $(n=1)$, inability to validate primary data sources $(n=2)$, results and conclusions bases on theoretical deduction and not field epidemiology data $(n=1)$.

The number or corrected articles on COVID-19 indicate the haste in publishing, which is not surprising considering the volume and the unprecedented interest of the research community and the public. In comparison to Ebola, as the other most recent public emergency of international concern, publication surge about COVID-19 resulted in comparable literature corrections.

Shorter time to correct or retract information in COVID-19 articles than those in Ebola articles may mean that both authors and editors behaved responsibly in ensuring the integrity of the published record and provide reliable evidence base to make valid decision in addressing the disease and the pandemic. On the other hand, the finding that 9\% of COVID-19 literature corrections were retractions, in comparison to none for articles on Ebola, indicates the pressure to publish may lead to research misbehavior.

This is just the first snapshot of the corrections to the research on COVID-19 pandemic. The pandemic continues (with more than 42 thousand published articles indexed in PubMed on August 20, 2020) and more corrections to the published literature are expected. We will be following them and assess how effective the scientific community was in ensuring reliable and valid evidence base for addressing the biggest global health emergency of this century.

As the COVID-19 pandemic continues, the research community should continue to adhere to the highest principles of research integrity in their work, the journals should continue to deal quickly and efficiently with correcting the published record, and bibliographical databases should consistently and rapidly record the corrections so that only valid evidence base can be used in health care. 
Funding: This study was funded by a grant to AM from the Croatian Science Foundation (Grant No. IP-2019-044882). The funder had no role in study design, data collection and analysis or decision for publication.

Authorship contributions: AM gave the idea for the study. JM, IB and AM developed the design of the study and search strategies. JM and AM identified the corrections and retractions to articles in PubMed. JM, IB and AM analyzed and interpreted the data. AM wrote the draft of the manuscript and JM and IB revised it for intellectual content. JM, IB and AM approved the final version of this manuscript and agree to be accountable for all aspects of the work in ensuring that questions related to the accuracy or integrity of any part of the work are appropriately investigated and resolved.

Competing interests: The authors declare no conflict of interest. Ana Marušić is the Co-editor in Chief of the Journal of Global Health.

1 World Health Organization. WHO announces COVID-19 outbreak a pandemic. [12 March 2020] . Available: https://www. euro.who.int/en/health-topics/health-emergencies/coronavirus-covid-19/news/news/2020/3/who-announces-covid19-outbreak-a-pandemic. Accessed July 22, 2020.

2 Palayew A, Norgaard O, Safreed-Harmon K, Andersen TH, Rasmussen LN, Lazarus JV. Pandemic publishing poses a new COVID-19 challenge. Nat Hum Behav. 2020;4:666-9. Medline:32576981 doi:10.1038/s41562-020-0911-0

3 Offord C. Lancet, NEJM retract Surgisphere studies on COVID-19 patients. The Scientist. Available: https://www.the-scientist.com/news-opinion/lancet-retracts-surgispheres-study-on-hydroxychloroquine-67613. Accessed July 22, 2020.

4 Bauchner H, Golub RM, Zylke J. Editorial concern - possible reporting of the same patients with COVID-19 in different reports. JAMA. 2020. Online ahead of print. Medline:32176775 doi:10.1001/jama.2020.3980

5 Glasziou PP. Waste in covid-19 research. BMJ. 2020;369:m1847. Medline:32398241

6 World Health Organization. Ebola outbreak in West Africa declared a public health emergency of international concern. 8 August 2014. Available: https://www.euro.who.int/en/health-topics/communicable-diseases/pages/news/news/2014/08/ ebola-outbreak-in-west-africa-declared-a-public-health-emergency-of-international-concern. Accessed July 22, 2020.

\section{Correspondence to:}

Prof. Ana Marušić, MD, PhD

University of Split School of

Medicine

Šoltanska 2

21000 Split, Croatia

ana.marusic@mefst.hr 